\title{
ILCEA
}

Revue de l'Institut des langues et cultures

d'Europe, Amérique, Afrique, Asie et Australie

37 | 2019

Des genres en Méditerranée : pratiques, représentations et transfert

\section{La bonne odeur : construction du genre dans le monde romain}

The Good Smell: Construction of the "Genre " in the Roman World

\section{Ana Maria Misdolea}

\section{(2) OpenEdition}

\section{Journals}

Édition électronique

URL : http://journals.openedition.org/ilcea/7639

DOI : 10.4000/ilcea.7639

ISSN : 2101-0609

Éditeur

UGA Éditions/Université Grenoble Alpes

\section{Édition imprimée}

ISBN : 978-2-37747-099-0

ISSN : 1639-6073

Référence électronique

Ana Maria Misdolea, "La bonne odeur : construction du genre dans le monde romain », ILCEA [En

ligne], 37 | 2019, mis en ligne le 04 novembre 2019, consulté le 15 janvier 2020. URL : http://

journals.openedition.org/ilcea/7639; DOI : 10.4000/ilcea.7639

Ce document a été généré automatiquement le 15 janvier 2020

(C) ILCEA 


\title{
La bonne odeur : construction $\mathrm{du}$ genre dans le monde romain
}

The Good Smell: Construction of the "Genre » in the Roman World

\author{
Ana Maria Misdolea
}

\section{Introduction}

1 Près de la colonie d'Hippone, qui est en Afrique sur le bord de mer, nous dit Pline le Jeune dans l'une de ses Lettres ${ }^{1}$, se trouve un étang navigable. Un dauphin, qui s'y était aventuré, était devenu ami avec un jeune garçon. Ils jouaient chaque jour ensemble dans l'eau, sous les yeux étonnés des badauds. Un jour, alors que le dauphin se reposait sur le sable avant de rejoindre l'onde, un certain Octavius Avitus fit répandre sur l'animal des parfums, qui le rendirent malade. Cette anecdote, rapportée par Pline le Jeune, montre que le parfum, fabriqué par la main de l'homme, s'il convient au genre humain, genus humanum, ou au genre divin, genus diuinum ${ }^{2}$, ne sied pas au genre animal, genus animale. L'olfaction dessine donc une frontière entre l'humain, l'animal et le divin, dont l'anecdote rapportée par Pline n'est qu'un exemple. Ce sont les frontières que trace l'odeur dans l'imaginaire des Romains ${ }^{3}$ que nous voudrions analyser ici, dans le champ du vivant mais également dans le champ social, notamment dans la distinction qui s'y opère entre l'homme et la femme, entre genus uirile et genus muliebre. Y a-t-il une «bonne odeur » (et inversement, une mauvaise odeur) qui contribuerait à définir la limite entre conformité et non-conformité à une norme ${ }^{4}$ Comment comprendre cette « bonne odeur », lorsque les domaines du social, de l'imaginaire et du sensoriel se croisent? Nous essaierons d'abord de mettre en évidence les systèmes d'oppositions sociales qui traversent le champ olfactif et qui participent à la construction d'une norme, puis nous verrons comment les normes dans le domaine olfactif contribuent à la construction du genre féminin. 


\section{Catégorisation et odeur}

2 Comment l'odeur intervient-elle dans la construction des normes sociales et dans les processus de catégorisation au sein de la société romaine?

Le vocabulaire olfactif latin, relativement pauvre, fonctionne selon un système d'oppositions. Les adjectifs qui qualifient l'odeur construisent ainsi des couples antithétiques. Les odeurs renvoient à ce qui est plaisant (iucundus) ou déplaisant (fredus ), suave (suauis) ou puant (putidus), à ce qui est doux (dulcis) ou âcre (acer), à ce qui est lourd, désagréable, oppressant (grauis) ou au contraire vif, pénétrant, léger (acutus). Ainsi toutes les odeurs ne sont-elles pas aptes à entrer dans la composition des parfums: Pline l'Ancien note que l'odeur grauis n'a pas sa place dans la recette d'un parfum ${ }^{5}$. Les images associées aux odeurs présentent elles aussi des connotations divergentes, qui tendent à rapporter l'odeur agréable à la fleur et à la divinité, et l'odeur désagréable à l'animal. Parmi les animaux, le bouc et le phoque par exemple sont évoqués lorsqu'il est question de mauvaises odeurs 6 . À l'inverse, lorsque Martial veut vanter la bonne haleine de la jeune Érotion, il évoque les productions de la nature (miel, ambre) et recourt notamment au référent végétal : Érotion « dont l'haleine avait le parfum d'une roseraie de Paestum ${ }^{7}$.»

3 Chez les philosophes "naturalistes ", dont le champ d'investigation s'étend à la natura des êtres vivants et non-vivants, l'existence de ces couples antithétiques trouve une explication physique. Il est vrai que les philosophes s'intéressent davantage à la vision (Aristote dit ainsi que «la vue est, de tous nos sens, celui qui fait acquérir le plus de connaissances $»^{8}$ ), mais ils développent également leur réflexion sur les autres sens de la perception. Même si les odeurs, plus que les autres sensations, sont évanescentes et difficiles à appréhender ${ }^{9}$, elles offrent un spectre large qui s'articule, du point de vue du sujet percevant, autour des pôles de l'attraction et de la répulsion. Après Théophraste, disciple d'Aristote, et son traité Sur les odeurs, le Romain Lucrèce s'intéresse à l'odorat, en le replaçant dans la théorie de la perception développée par Épicure : les simulacra (le mot traduit le grec č̌ $\delta \omega \lambda \alpha)$ émanent des objets et pénètrent en nous par des canaux (foramina) jusqu'aux organes de la sensation (les narines pour l'odorat). On retrouve un système d'opposition : les atomes qui composent les simulacra sont lisses et ronds (leuis et rotundus) ou crochus et serrés (hamatus et asper), et c'est cette différence qui explique qu'une odeur est ressentie comme agréable ou désagréable ; il y a aussi des atomes ni tout à fait lisses, ni tout à fait crochus et armés de pointes, plus propres à chatouiller les sens qu'à les blesser ${ }^{10}$. Le philosophe romain ne manque pas de mettre en évidence la relativité des appréciations sur les odeurs, qui réapparaitra un siècle et demi plus tard dans l'anecdote de Pline le Jeune que nous avons mentionnée en introduction : Lucrèce précise que la marjolaine met en fuite le pourceau, qui déteste toute espèce de parfums, car ils sont pour lui du poison, alors que l'homme y trouve une source de vie. Les feuilles d'olivier sauvage déplaisent à l'homme, mais elles sont appréciées de la chèvre comme s'il s'agissait d'ambroisie ou de nectar, c'est-à-dire les substances qui plaisent aux dieux ${ }^{11}$.

Or cette relativité, fondée sur la distinction entre hommes et animaux, que nous retrouvons dans le monde romain dans l'ouvrage de Lucrèce qui repense dans une perspective épicurienne l'argumentation des milieux sceptiques grecs ${ }^{12}$, se superpose aux discours populaires sur les odeurs, plus qu'elle ne la remet en question. 
Dans la société romaine, les odeurs renvoient en effet le plus souvent à des phénomènes de catégorisations et de hiérarchisations. Le théâtre comique, de ce point de vue, nous donne en partie accès aux représentations et aux mentalités. Lorsque les deux esclaves Grumion et Tranion s'insultent, dans la comédie La Maison hantée composée par Plaute au début du II siècle av. J.-C., l'odeur qui caractérise chacun d'entre eux les place sur une échelle sociale : parmi les esclaves, le fermier Grumion, vivant dans des conditions plus modestes, est miser, "malheureux ", car il sent l'ail et plus généralement toutes sortes d'odeurs rustiques : «Quelle pure saleté tu fais, rustre, bouc, étable à cochons, mélange de fiente et d'ordure ", lui dit Tranion, alors que Tranion lui-même, fortunatus (« qui a de la chance »), sent les parfums exotiques (unguenta exotica) et mange des plats fins (facetis uictibus ${ }^{13}$ ). L'évocation de l'odeur s'intègre en effet dans un jeu de hiérarchie sociale, comme le rappelle Stevens (2008). L'odeur est ainsi un marqueur de l'origine ou du niveau social. Il y a par conséquent un lien fort entre la perception de l'odeur et ce que Stevens appelle la " cognition sociale ", c'est-à-dire l'expression d'une pensée sur les interactions sociales. On trouve là une forme de savoir, fondé sur la connaissance de la norme. Les oppositions qui se dessinent concernent la ville et la campagne, mais également le luxe venu de l'Orient (unguenta exotica) et la rusticité de l'indigène. Le jeu comique de Plaute, certes fondé sur l'exagération, met cependant en lumière, comme par un effet de loupe, des lignes de démarcation entre différentes catégories sociales.

Il faut cependant tenir compte de la complexité des critères de catégorisation. La valorisation apparente des unguenta exotica est à nuancer par l'interdiction de leur vente à Rome décidée par les censeurs en 189 av. J.-C., interdiction à laquelle devaient penser les spectateurs de La Maison hantée. Les censeurs redoutaient de fait l'invasion du luxe étranger. Cette crainte crée des tensions, à l'intérieur même de la société romaine, qui viennent compliquer le jeu de catégorisation, comme en témoigne le débat houleux concernant l'abrogation, en 195 av. J.-C., de la loi Oppia qui limitait l'étalage des richesses chez les matrones romaines ${ }^{14}$.

7 On comprend dès lors aisément que la «bonne odeur " n'est pas nécessairement celle qui produit une sensation agréable. Elle met en jeu un jugement esthétique, mais elle peut aussi mettre en jeu un jugement moral. Par ailleurs, la catégorie du «bon » dans le domaine des senteurs est variable et soumis aux modes, comme le rappelle Pline l'Ancien dans l'Histoire naturelle ${ }^{15}$. Enfin, le critère du convenable et de l'approprié, l' aptum, détermine la grille de ce qui est valorisé ou déprécié, dans les rapports complexes entre odeurs et pratiques sociales et culturelles. Alors que le bois d'encens, rare pour les Romains, est réservé aux autels des dieux, les Sabéens, peuple d'Arabie, s'en servent pour cuire leurs aliments, et vont jusqu'à brûler du styrax pour se débarrasser de l'odeur du bois d'encens ${ }^{16}$. La définition de la «bonne odeur » repose donc sur un réseau complexe de pratiques et de facteurs sociaux, traversés par la notion de norme.

\section{Femme et odeur}

8 Comment ces systèmes d'opposition interviennent-ils dans la catégorie du féminin? Qu'en est-il des relations entre la femme et l'odeur?

9 Si «avoir l'odorat fin » n'est pas l'apanage des femmes (chez le satiriste Martial, est nasutus celui « qui a du flair»), force est de constater que ce sont souvent elles qui sont associées à la sagacité. Trouver la «bonne » odeur, c'est porter un jugement vrai et 
assuré : il s'agit d'être capable de reconnaître ce qu'on sent. Ainsi dans la langue latine, l'adjectif sagax, «à l'odorat fin » - comme les chiens, dit Cicéron - a pris un sens figuré pour désigner un esprit subtil qui devine les choses. Praesagire, c'est " avoir du flair». Cicéron parle ainsi des vieilles femmes : « sagire, c'est avoir un sens aigu, du flair : d'où les vieilles appelées sagae, parce qu'elles prétendent savoir beaucoup de choses, et les chiens, sagaces. Celui donc qui "sent" une chose avant qu'elle ne se présente, on dit de lui qu'il présage, c'est-à-dire qu'il prédit l'avenir. Il y a donc dans les âmes une aptitude à présager ${ }^{17}$. On notera que le rapprochement entre le chien et la vieille femme n'est pas tout à fait flatteur. Certes, le chien identifie des odeurs, mais il n'a pas de pouvoir autre ; il n'a pas de prise sur l'odeur et ne peut la manipuler. Il est dans la passivité, de ce point de vue. Par ailleurs, l'image de la femme-chienne renvoie à un cliché misogyne que l'on trouvait déjà chez les Grecs. Sémonide d'Amorgos (VII siècle av.J.-C.) comparait les types de femmes à différents animaux. La femme-chienne, hystérique, se laisse aller à la passion. La femme-cavale, elle, est dans l'apparence séductrice : elle passe sa journée à se laver et à se parfumer. Ainsi l'odorat et l'odeur contribuent-ils, entre autres critères, à rejeter la femme du côté de l'animalité.

Sur le plan éthique, se dégagent deux images antagonistes de la «bonne odeur » chez la femme romaine, celle du parfum qui contribue à la séduction, et celle, a contrario, de l'absence, valorisée, d'odeur artificielle. Dans le premier cas, la femme se trouve associée au parfum, parce que l'agréable devient l'équivalent du désirable. Le parfum est lié à l'amour. Mais de quel amour s'agit-il ? Dans la comédie de Plaute intitulée Casina, les fragrances évoquées par les noms des personnages féminins suggèrent divers types d'attirance amoureuse (Connors, 1997) : la jeune esclave «Casina » tire son nom de la cassia (cannelle), épice parfumée de l'Orient importée à Rome par l'Afrique ou l'Arabie. C'est de Casina qu'est amoureux le vieillard de la pièce. Mais tout comme les parfums exotiques ne conviennent pas aux vieillards, la jeune Casina échappera au vieillard Lysidame à la fin de la pièce. Le nom de "Pardalisca", la servante de la matrone, l'épouse du vieillard, évoque la panthère. L'haleine de la panthère était censée posséder un pouvoir d'attraction ${ }^{18}$; une scène de rapprochement accidentel entre le vieillard et Pardalisca évoque cette séduction olfactive ponctuelle. Enfin «Myrrhina », le nom de la voisine de la matrone, matrone elle-même, vient de myrrha (l'arbre à myrrhe), que l'on met en rapport avec Vénus, le désir sexuel et la réconciliation au sein du mariage, qui annonce de fait le dénouement de la pièce ${ }^{19}$. Les parfums sont ainsi révélateurs des relations symboliques entre les types de personnages (vieillard, servante, matrone). Dans un passage de La Maison hantée, on parle d'un parfum associé à la sueur, et ce mélange, comparé aux sauces que font les cuisiniers, révèle la vieille courtisane : "vois ces vieilles qui se parfument de toutes sortes de parfums, qui tâchent de se retaper; décrépites, édentées, elles veulent sous une couche de fard cacher les défauts de leur personne; mais quand le relent de la sueur se mêle à leurs parfums, l'odeur qu'elles dégagent alors ressemble à ces mélanges de sauces que font parfois les cuisiniers. On ne sait ce qu'elles sentent, sinon qu'elles sentent mauvais ${ }^{20}$.»

11 Mais si la vieille courtisane est repoussante lorsqu'elle se parfume, ce n'est pas le cas de la jeune courtisane. L'âge est donc bien discriminant. Les fragrances conviennent à la jeune courtisane, qui en use pour son corps mais aussi dans sa maison. Dans les Ménechmes de Plaute, la courtisane Érotie, préparant un banquet pour son amant, demande à ses esclaves de garnir les lits et de brûler des parfums : la parure est un appât pour les amants (v. 353-355). Les « bonnes odeurs » sont associées au banquet, où 
dominent le luxe ostentatoire et le plaisir sensuel. Le banquet et les parfums qui y sont répandus font partie du "vivre à la grecque " (pergraecari) : couronnes de fleurs, vins dans lesquels on a versé des parfums, sont là pour créer le plaisir olfactif.

Le parfum participe donc, bien évidemment, de la séduction féminine. Quand une femme qui s'est parfumée passe, l'odeur qu'elle répand attire même ceux qui sont occupés à autre chose ${ }^{21}$. Le parfum est un élément de la parure féminine, au même titre que le maquillage. Mais si la femme se pare pour attirer l'homme dans le jeu de séduction, Ovide nous dit qu'elle se pare aussi pour se plaire à elle-même, dans le traité Sur les cosmétiques. Après avoir donné une recette pour un fard, il en donne une autre pour un parfum. L'artifice, qu'il soit maquillage ou parfum, est donc ambivalent: il s'adresse tout autant à soi-même qu'à autrui. Le recours à l'art de la parfumerie est recommandé pour plaire, mais le référent reste la nature. Les baisers du jeune Diadumenus sont comparés par Martial au parfum «que dégage un fruit entamé par une délicate bouche de jeune fille », ou encore à celui " qu'apporte la brise qui a passé sur le safran de Corycos $\aleph^{22}$. La nature se charge alors d'érotisme olfactif. Si Théophraste fait ce constat qu'aux femmes conviennent bien les parfums forts et persistants, comme le nard, l'huile de myrrhe staktè, ou encore le parfum appelé megaleion (mélange de différentes senteurs, dont la cannelle) ${ }^{23}$, il semble qu'il n'y ait pas eu à proprement parler de senteurs réservées aux femmes. La femme se pare de ce que la nature lui offre comme «bonnes odeurs » pour se rendre désirable.

13 Au cours du temps, la propension des Romains au luxe et au parfum ira en s'accentuant. Pline l'Ancien souligne en effet que le parfum a une histoire. Au temps de la guerre de Troie, il n'existait pas, nous dit le naturaliste ${ }^{24}$. C'est aux Perses qu'il faut l'attribuer : ils s'en inondaient pour étouffer la mauvaise odeur due à leur malpropreté. Par la suite, après les Grecs qui commencèrent à recourir eux aussi aux parfums, l'usage s'en répandit à Rome, « où la jouissance qu'il procure a été rangée parmi les biens de la vie les plus recherchés, et même les plus distingués ${ }^{25}$. " Pline l'Ancien condamne l'évolution dans l'utilisation des parfums et en particulier les usages de ses contemporains de la fin du $\mathrm{I}^{\mathrm{er}}$ siècle av. J.-C. Ce ne sont pas les seules femmes qui usent et abusent des parfums, mais les hommes aussi. Néron s'enduisait la plante des pieds de parfums, d'autres en aspergent les murs des bains. Le parfum est l'objet du luxe le plus inutile qui soit, parce qu'il est proprement éphémère. Le naturaliste se fait volontiers moraliste : même les étendards à l'armée sont désormais parfumés les jours de fête !

14 La dépravation qu'accompagne le recours excessif au parfum ne concerne donc pas seulement la femme qui, corrompue, peut aller jusqu'à commettre l'adultère ${ }^{26}$. La dépravation morale guette en réalité l'homme aussi et peut remettre en cause sa virilité. Martial décrit à merveille la peau brunie par les onguents chez les hommes efféminés, ou leurs cheveux enduits de parfums : «Charmenion [...] tu te promènes avec ta chevelure brillante de parfums [...] tes membres sont lisses grâce à la pâte épilatoire quotidienne [...] Cesse donc de m'appeler ton frère, si tu ne veux, Charmenion, que je t'appelle ma sœur ${ }^{27}$. " Le recours au parfum fait donc partie de l'accusation d'effémination. La transformation exécrable de l'homme en femme passe entre autres par l'adoption du parfum, qui brouille les frontières entre les genres.

Face à ce danger d'effacement des frontières, la deuxième association entre la femme et l'odeur qui se dessine dans l'imaginaire romain est celle de l'absence d'odeur particulière, qui préserve l'odeur naturelle propre à la femme. Avec la préservation de l'odeur naturelle, on entre alors, paradoxalement, dans la sphère du prescriptif. Le 
banquet par exemple n'est pas un lieu où doit se trouver la femme honorable, parce qu'il est souvent synonyme de lieu de débauche ${ }^{28}$. S'adonner à la boisson n'est bien sûr pas décent pour une femme, et la pratique du «test-haleine » permettait, selon Caton l'Ancien, de s'assurer de la moralité de la femme. Le baiser qu'elle donnait à ses parents servait en effet d'épreuve : si elle avait bu du vin, l'odeur la trahissait ${ }^{29}$. Mais surtout, la meilleure odeur pour la femme respectable est de ne rien sentir, précisément. Une vieille servante de comédie affirme : mulier recte olet, ubi nihil olet, « une femme sent convenablement quand elle ne sent rien ${ }^{30}$.» On retrouve le même type de propos dans le monde grec. Ainsi Xénophon fait-il dire à Socrate: "de même que la beauté d'un vêtement de femme n'est pas celle d'un vêtement d'homme, ce sont des odeurs différentes qui conviennent à l'homme et à la femme. Aucun homme en effet, n'est-il pas vrai? ne s'oint de parfum pour un autre homme. Quant aux femmes, surtout lorsque ce sont des jeunes mariées, comme celles de Nikératos et de Critobule ici présents, qu'ont-elles encore besoin de parfum? Elles en exhalent elles-mêmes ${ }^{31}$.» Certes, l'odeur qui convient à l'homme grec est l'odeur d'huile d'olive des gymnases, poursuit Socrate, et l'on fera remarquer que ce n'est pas l'usage à Rome; mais pour ce qui est de la jeune femme, la prescription morale est la même, car la jeune femme en particulier doit respecter l'odeur naturelle. Sa plus belle parure est sa jeunesse féconde.

\section{Divinités féminines et odeurs}

16 La complexité des rapports entre la femme et l'odeur, entre attraction et répulsion, pratique et idéal, réel et imaginaire, se retrouve chez les divinités féminines qu'honorent les Romains. On peut, en effet, comprendre la catégorie du féminin chez les divinités comme une projection de la division des sexes humains (Pirenne-Delforge, 2001).

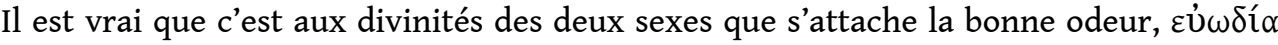
en grec $^{32}$, suauitas en latin. Lorsque paraît la divinité, qu'elle soit masculine ou féminine, son épiphanie se traduit, nous dit-on, par une lumière étincelante, mais aussi par la propagation d'un parfum délicieux. Les dieux se nourrissent d'ailleurs de nectar et d'ambroisie, mythiques nourritures parfumées qui participent à leur immortalité.

Mais ce sont les divinités féminines qui sont le plus volontiers décrites sous l'angle olfactif, souvent dans la séduction - notamment quand elles se parent, comme Héra pour séduire Zeus ${ }^{33}$. À Rome, une déesse est particulièrement liée aux bonnes odeurs végétales : lorsque Flora, la déesse des fleurs, parle, « sa bouche exhale l'odeur des roses printanières ", nous dit le poète Ovide ${ }^{34}$.

Nous n'entrerons pas ici dans la question de l'utilisation des senteurs dans le rite religieux, qui dépasserait le cadre de ce travail, mais nous voudrions souligner quelques aspects surprenants du rapport de deux déesses à l'odeur. La première est Vénus. À la déesse du désir, et plus particulièrement de la sexualité dans le mariage, sont liés la rose et le myrte ${ }^{35}$. Mais Vénus avait un sanctuaire près de la Cloaca Maxima, le "grand égout " de Rome, datant de l'époque de Tarquin l'Ancien, qui drainait les eaux du Forum romain. Pourquoi un temple si près des odeurs nauséabondes, pour la déesse des roses et de l'amour? Ce sont les chrétiens qui vont souligner ce contraste, en évoquant le temple de la Vénus "Cloacina", c'est-à-dire "du cloaque ». À en croire Pline l'Ancien, la fondation de son sanctuaire aurait été liée à un rite de purification : après l'enlèvement des Sabines, qui devinrent les premières épouses des Romains, il fallait 
effectuer une purification avec du myrte à cet endroit ${ }^{36}$. Le récit étiologique associe ainsi purification et mariage à la localisation du sanctuaire de Vénus près d'un endroit sentant mauvais.

Encore plus curieux est le cas de Méfitis, déesse des odeurs pestilentielles. Divinité italique, elle possédait aussi un lieu de culte sur la colline de l'Esquilin, à Rome. Pourquoi honorer une déesse des mauvaises odeurs? Pourquoi s'agit-il là d'une déesse et non d'un dieu? Sa féminité est-elle significative?

"Mefitis ", en tant qu'appellatif, désigne la mauvaise odeur, la puanteur, putor ou fœetor,

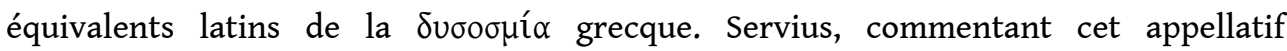
"Mefitis ", indique qu'il s'agit de la puanteur qui se dégage des eaux sulfureuses ${ }^{37}$. La mauvaise odeur est celle de l'altération de l'air, tandis que la bonne odeur se trouve là où l'air n'est pas altéré. Méfitis est ainsi la dea odoris grauissimi, la déesse de l'odeur nauséabonde en général. Elle est donc maléfique et il faut se la concilier, comme on tente de se concilier par des rites propitiatoires Robigo, la "rouille", maladie des céréales (la nielle), ou Febris, la "fièvre", divinités que les Romains invoquent pour préserver, précisément, la bonne santé des récoltes et du corps humain. Ces divinités maléfiques, comme Febris ou Mala Fortuna, possédaient leur lieu de culte sur l'Esquilin.

Or la déesse Méfitis changea de fonction lorsqu'elle passa de l'univers culturel italique à l'univers proprement romain. Poccetti propose de voir dans son changement de fonction un exemple du processus d'interpretatio romaine (transformation de la nature divine désignée par un théonyme au moment du passage d'un panthéon à un autre, Poccetti, 1982 : 243). Les Romains sélectionnèrent en effet comme trait définitoire de la nature de la divinité italique Méfitis une caractéristique de ses lieux de culte, les exhalaisons malodorantes. Méfitis devint ainsi, dans le panthéon romain, la divinité de la mauvaise odeur.

23 Mais qu'était-elle à l'origine? Les étymologies antiques rapprochent son nom du grec

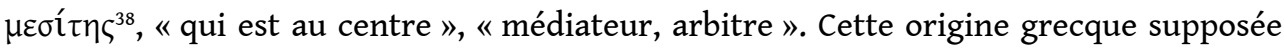
explique la graphie ph, due sans doute aux copistes et reprise dans les éditions modernes: "méphitique » signifie ainsi pour nous « dont l'exhalaison est malfaisante et toxique ». Une proposition moderne de reconstruction lie le nom Méfitis à l'indoeuropéen *medh-, "enivrer", qui s'expliquerait par le fait que les exhalaisons sulfureuses font perdre leurs moyens à ceux qui les respirent. Dans l'Antiquité, l'eau de certaines sources était réputée avoir le même effet que le vin. Méfitis serait ainsi une «force enivrante ».

Poccetti rapproche cette proposition d'étymologie des analyses de Dumézil qui présente l'ivresse comme partie intégrante du concept de souveraineté dans l'idéologie indo-européenne, pouvant s'incarner dans un être de sexe féminin, comme la reine Medb dans la mythologie celtique irlandaise, ou Madhavi, épouse royale dans l'épopée indienne (Dumézil, 1971 : 341). De fait, les lieux de culte italiques qui ont livré du matériel en rapport avec Méfitis semblent l'associer à Jupiter ${ }^{39}$; Jupiter et Méfitis constitueraient ainsi le couple divin souverain. Méfitis est mise en rapport avec la terre, l'eau et les sources (se substituant sans doute à un culte chtonien préexistant) ; or les divinités de l'eau se voyaient accorder une place plus importante dans la religion italique que dans la religion romaine. Les sources aux propriétés géophysiques remarquables, comme le sont les exhalaisons de soufre, constituaient donc des lieux de culte privilégiés pour la divinité Méfitis. Les Romains ne retinrent que l'idée d'un lieu de culte à l'odeur nauséabonde, puis celle de la mauvaise odeur. Virgile évoque ainsi la 
mefitis en tant qu'exhalaison pestilentielle à propos de la consultation d'un oracle près d'une source sacrée "qui résonne dans l'immensité de la forêt, et qui exhale dans l'obscurité une sauvage odeur de soufre ${ }^{40} »$. Cette source sera plus tard personnifiée sous les traits d'une Sibylle, Albunée. Ainsi la « mauvaise odeur » de la divinité féminine dans le panthéon romain, puissance mystérieuse, renvoie in fine à des cultes anciens, liés à la terre et à l'eau, mais dont le souvenir s'est perdu. Avec cet oubli, les valeurs se sont inversées, conférant à l'entité féminine une valeur olfactive négative entraînant la répulsion, mais également une valeur oraculaire, comme en témoigne Virgile.

\section{Conclusion}

L'odeur apparait ainsi chez les Romains comme un trait discriminant, qui participe à l'élaboration spontanée des frontières entre les catégories naturelles et sociales du monde. L'odeur dit l'identité. Le divin, l'humain, l'animal et le végétal comportent des odeurs qui les distinguent. Les auteurs latins sont cependant sensibles à la relativité des notions de plaisir et de convenance en matière d'odeur. L'idéal de la vertu vient compliquer encore le jeu de la hiérarchisation.

Cette complexité de l'odeur comme critère apparaît aussi lorsqu'elle sert à distinguer le genre féminin du genre masculin. La "bonne odeur ", c'est celle du parfum, parure qui contribue à la séduction. Or le fait de se parfumer « est dans l'Antiquité une pratique sociale et comportementale normative ", comme le soulignent Bodiou et Mehl (2011: 103). Se parfumer, pour un homme, fait courir le risque de l'effémination. Mais la bonne odeur de la femme, c'est aussi la bonne odeur de la nature, la féminité étant alors envisagée tout orientée vers le mariage, comme le montre aussi la féminité dans la divinité, qui allie fécondité, souveraineté et purification.

\section{BIBLIOGRAPHIE}

Bodiou Lydie \& MeHL Véronique [dir.] (2011), Odeurs antiques, Paris : Les Belles Lettres.

BODIOU Lydie, FRèRE Dominique \& MEHL Véronique [dir.] (2008), Parfums et odeurs dans l'Antiquité, Rennes : Presses universitaires de Rennes.

BRUn Jean-Pierre \& FERNANDEZ Xavier [dir.] (2015), Parfums antiques : de l'archéologue au chimiste, Grasse : Musée international de la parfumerie.

Classen Constance, Howes David \& SYNNOTT Anthony [dir.] (1994), Aroma: the Cultural History of Smell, Londres - New York : Routledge.

ConNors Catherine (1997), « Scents and Sensibility in Plautus' Casina », The Classical Quarterly, 47, 305-309.

Courtil Jean-Christophe, Courtray Régis \& Allely Annie [dir.] (2018), Goûts et odeurs dans

l'Antiquité, revue Pallas, 106. 
DEONNA Waldemar (2003), Euôdia. Croyances antiques et modernes. L'odeur suave des dieux et des élus, Turin : Nino Aragno. (Ouvrage original publié en 1939 sous le titre « Euôdia. Croyances antiques et modernes. L'odeur suave des dieux et des élus », Genava, 17, 167-262.)

DuMÉzIL Georges (1971), Mythe et épopée II, Paris : Gallimard.

FAURE Paul (1996), Parfums et aromates de l'Antiquité, Paris : Hachette.

FEDELI Paolo (2005), Écologie antique : milieux et modes de vie dans le monde romain (I. Cogitore, trad.), Dijon : Darantière. (Ouvrage original publié en 1990 sous le titre La natura violata. Ecologia e mondo romano, Palerme : Sellerio.)

ITGENSHORST Tanja \& LE Doze Philippe [dir.] (2017), La norme sous la République et le Haut-Empire romain : élaboration, diffusion et contournements, Bordeaux : Ausonius.

LEJEUnE Michel (1972), « Inscriptions de Rossano di Vaglio 1972 », Rendiconti dell'Accademia dei Lincei, 27, 399-414.

LILJA Saara (1972), The Treatment of odours in the poetry of Antiquity, Helsinki : Societas scientiarum Fennica.

Poccetti Paolo (1982), « Mefitis », AION, 4, 237-260.

PirenNe-Delforge Vinciane (2001), « Prairie d'Aphrodite et jardin de Pandore », É. Delruelle \& V. Pirenne-Delforge (dir.), Kepoi : de la religion à la philosophie; mélanges offerts à André Motte, Liège : Centre International d'Étude de la Religion Grecque Antique, 83-99.

SQUILLACE Giuseppe (2015), Le Lacrime di Mirra: miti e luoghi dei profumi nel mondo antico, Bologne : Il Mulino.

STEVENS Benjamin (2008), « The Scent of Language and Social Synaesthesia at Rome », The Classical World, 101, 159-171.

\section{NOTES}

1. Pline le Jeune, Lettres (IX, 33). Il s'agit de la lagune de Bizerte. Pline l'Ancien, son oncle, livre également une version de cette anecdote dans son Histoire naturelle (IX, 26).

2. Ce type d'onction concernait surtout les statues de culte: c'est par superstition qu'agit Octavius Avitus, étonné devant un tel prodige, qu'il prend pour une manifestation du divin.

3. La question des odeurs a fait l'objet de nombreux travaux ces dernières années, même si le domaine romain a été moins étudié que le domaine grec. Citons notamment, sans prétention à l'exhaustivité : Bodiou, Frère \& Mehl (2008) ; Brun \& Fernandez (2015) ; Classen, Howes \& Synnott (1994) ; Courtil, Courtray \& Allely (2018); Faure (1996) ; Lilja (1972); Squillace (2015).

4. Sur la notion de norme plus généralement, voir Itgenshorst \& Le Doze (2017).

5. Sur la composition des parfums, voir notamment Fedeli (2005).

6. Un système de représentations analogue est présent chez les Grecs, pour qui « l'odeur est un moyen privilégié pour comprendre le fonctionnement du corps humain mais aussi pour classifier leur univers. Si les dieux embaument naturellement, les hommes sont olfactivement neutres, tandis que les animaux puent » (Bodiou \& Mehl, $2011: 226$ ).

7. Martial, Épigrammes $(V, 37,9)$ : fragrauit ore quod rosarium Paesti. Nous empruntons nos traductions aux éditions de la Collection des Universités de France.

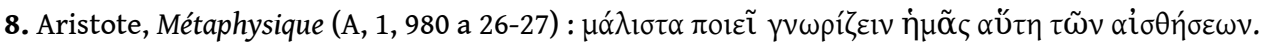

9. Lucrèce, Sur la nature (IV, 673-705).

10. Lucrèce, Sur la nature (II, 398-430). Voir Épicure, Lettre à Hérodote (53). 
11. Lucrèce, Sur la nature (VI, 970-975). Ainsi, «telle odeur convient mieux à telle créature, suivant les différentes espèces ", aliis alius magis est animantibus aptus / dissimilis propter formas (IV, 677).

12. Le premier «trope » sceptique, parmi les dix tropes attribués au philosophe grec Énésidème ( ${ }^{\text {er }}$ siècle av. J.-C.), rappelle qu'à cause de la différence entre les animaux, les mêmes choses ne produisent pas les mêmes sensations d'une espèce à l'autre.

13. Plaute, La Maison hantée (41 et suiv.).

14. Tite-Live, Histoire romaine (XXXIV, 1, 1 et suiv.). Face à Caton qui s'oppose à l'abrogation, Lucius Valerius rappelle que les ancêtres respectaient le mundus muliebris, la «parure des femmes ", qui consistait en munditiae et ornatus et cultus (élégances, ornements et soins) : ce sont là les distinctions, insignia, propres aux femmes (XXXIV, 7, 9).

15. Pline l'Ancien, Histoire naturelle (XIII, 2) : «à l'égard des parfums souvent la mode et la faveur ont changé. Dans l'antiquité, le plus estimé était le parfum de l'île de Délos ; plus tard ce fut celui de Mendès : ces variations ne sont pas dues seulement aux mélanges et aux proportions; mais les mêmes sucs sont en faveur ou défaveur suivant les lieux, et suivant l'amélioration ou la dégénération des substances. Le parfum d'iris de Corinthe a longtemps eu la vogue, puis celui de Cyzique. Il en a été de même pour le parfum de roses de Phasélis, prééminence qui fut enlevée par Naples, Capoue, Préneste. »

16. Pline l'Ancien, Histoire naturelle (XII, 40).

17. Cicéron, Sur la divination (I, 65-66). Le terme sapientia, "sagesse », est issu de sapere dont le champ sémantique englobe les sens propres " avoir du goût », " exhaler une odeur » ou " sentir quelque chose par le sens du goût», et les sens figurés "avoir du discernement» et « comprendre, savoir ».

18. Aristote, Histoire des animaux (612 a 12-15). C'est le seul animal qui n'a pas de mauvaise odeur, tout au contraire.

19. Plaute s'inspire sans doute d'une autre Myrrhine de comédie, celle de l'épouse de Cinésias dans la Lysistrata d'Aristophane : l'épouse parfume son mari, promesse des ébats sexuels à venir (Lysistrata, 930 et suiv.).

20. Plaute, La Maison hantée (274-278) : Nam istae ueteres, quae se unguentis unctitant, interpoles, / Vetulae, edentulae, quae uitia corporis fuco occulunt, / Vbi sese sudor cum unguentis consociauit, ilico / Itidem olent quasi cum una multa iura confudit cocus : / Quid olant nescias, nisi id unum, ut male olere intellegas.

21. Pline l'Ancien, Histoire naturelle (XIII, 4).

22. Martial, Épigrammes (III, 65, 2) : Quod spirat tenera malum mordente puella, / quod de Corycio quae uenit aura croco.

23. Théophraste, Sur les odeurs (42): aux hommes conviennent, selon lui, des senteurs plus légères, comme le parfum de rose ou de lys.

24. Pline l'Ancien, Histoire naturelle (XIII, 1-4), qui précise cependant qu'apparaît déjà dans l'Iliade une huile fleurant la rose. Voir également Ovide, Fastes (I, 338-348) pour les premiers usages des parfums dans les rites religieux.

25. Pline l'Ancien, Histoire naturelle (XIII, 1): uoluptas eius [...] inter lautissima atque etiam honestissima uitae bona admissa est. C'est généralement des guerres du début du II ${ }^{\mathrm{e}}$ siècle av. J.-C. qu'on date l'ouverture des Romains au luxe étranger. Voir Squillace (2015 : 78 et suiv.).

26. Voir en particulier l'attaque en règle de Juvénal contre les femmes de son époque dans sa célèbre satire sur les femmes: la matrone romaine achète les essences importées d'Inde pour paraître belle à son amant (Satires, VI, 465-466).

27. Martial, Épigrammes $(X, 65)$ : Charmenion... tu flexa nitidus coma uagaris / ... leuis dropace tu cotidiano / ... Quare desine me uocare fratrem, / ne te, Charmenion, uocem sororem.

28. L'avocat Cicéron, lorsqu'il veut brosser le portrait-charge des partisans de Catilina, les décrit au banquet: «Ah! je les vois : ils sont couchés autour de leurs tables, tenant dans leurs bras des 
femmes sans pudeur, alanguis de vin, gorgés de nourriture, la tête ceinte de fleurs, le corps oint de parfums, vidés par la débauche » (Deuxième Catilinaire, $V, 10-11$ ).

29. Pline l'Ancien, Histoire naturelle (XIV, 14). On notera que les coutumes de l'époque de Caton l'Ancien, idéalisée dans les siècles suivants, sont constamment remises en question avec toute la fougue dont la jeunesse est capable. Chez Martial, un jeune homme s'emporte ainsi contre son précepteur: «Ai-je revêtu un vêtement de pourpre ou parfumé ma chevelure ? Tu t'exclames : "Jamais ton père n'avait fait cela!" Assez! Je ne peux souffrir un affranchi qui fait le Caton " ( Épigrammes, XI, 39 : Si Tyrios sumpsi cultus unxiue capillos, / exclamas " Numquam fecerat ista pater »; / Desine; non possum libertum ferre Catonem). De même, Martial rapporte que les femmes de son époque se servent des pastilles du parfumeur Cosmus pour masquer leur haleine vineuse ( Épigrammes, II, 87). Commentant la phrase de Plaute sur la femme qui n'a aucune odeur, Squillace note que c'était déjà, au temps de Plaute, une : « pura utopia di fronte a una società in cambiamento o già irrimediabilmente mutata. » $(2015: 85)$

30. Plaute, La Maison hantée (273).

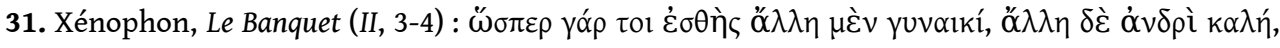

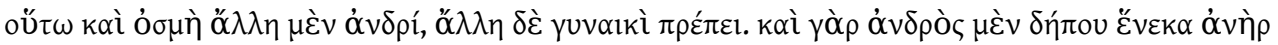

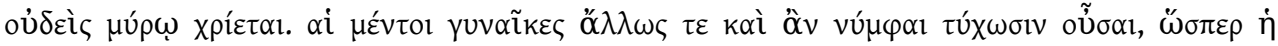

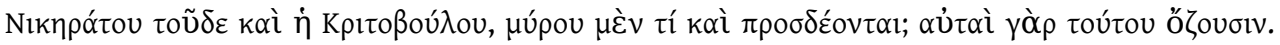
L'interlocuteur de Socrate finit par lui demander quelle odeur convient aux citoyens qui n'ont plus l'âge d'aller au gymnase ; « celle de la vertu », répond Socrate...

32. Voir sur ce sujet Deonna, 2003 [1939].

33. Homère, Iliade (XIV, 170-177).

34. Ovide, Fastes $(V, 194)$ : uernas efflat ab ore rosas.

35. L'Aphrodite grecque, née dans l'« odorante Chypre " (Hymne Homérique à Aphrodite), est elle aussi liée aux fleurs et aux parfums.

36. Pline l'Ancien, Histoire naturelle (XV, 119-120).

37. Servius, Commentaire à l'Énéide de Virgile (VII, 84).

38. Priscien (Grammaire, III, 328, 5) trouve sans doute cette étymologie chez Varron. Voir Poccetti (1982).

39. C'est notamment le cas à Rossano di Vaglio ; voir Lejeune (1972: 405).

40. Virgile, Énéide (VII, 84) : nemorum quae maxima sacro / fonte sonat saeuamque exhalat opaca mephitim.

\section{RÉSUMÉS}

Dans cet article, l'odeur est envisagée comme un élément participant, au sein de la société romaine, à l'élaboration d'une norme, mais aussi plus particulièrement à la construction du genre féminin. Se dégagent deux images antagonistes de la "bonne odeur " chez la femme romaine, celle du parfum qui contribue à la séduction, et a contrario celle de l'absence, valorisée, d'odeur artificielle. Dans son rapport à l'odeur, le divin féminin, projection de l'humain, met en valeur souveraineté, purification et fécondité.

In this paper, smell is considered as an element involved in the development of a norm within the Roman society and more particularly in the construction of the feminine gender. There are two antagonistic images of the "good smell" in the Roman woman, that of perfume, which is seen 
as a major attribute of seduction, versus the much praised absence of artificial smell. The feminine divine, as a projection of the human, is based on sovereignty, purification and fertility.

INDEX

Keywords : smell, perfume, gender, society, woman, proper, norm, Romans

Mots-clés : odeur, parfum, genre, société, femme, convenable, norme, Romains

\section{AUTEUR}

\section{ANA MARIA MISDOLEA}

Paris-Sorbonne

Équipe d'Accueil 4081 « Rome et ses renaissances » 